\title{
Efficacy and Safety of a Decision Support Intervention for Basal Insulin Self-Titration Assisted by the Nurse in Outpatients with T2DM: A Randomized Controlled Trial
}

This article was published in the following Dove Press journal:

Diabetes, Metabolic Syndrome and Obesity: Targets and Therapy

\author{
Xiling $\mathrm{Hu} \mathbb{( D}^{1}{ }^{1} *$ \\ Hongrong Deng $\mathbb{D}^{2, *}$ \\ Yao Zhang ${ }^{2}$ \\ Xiaodi Guo ${ }^{2}$ \\ Mengyin $\mathrm{Cai}^{2}$ \\ Cong Ling ${ }^{3, *}$ \\ Kun $\mathrm{Li}^{4, *}$
}

\begin{abstract}
'Department of Medicine, The Third Affiliated Hospital of Sun Yat-sen University, Guangzhou, People's Republic of China; ${ }^{2}$ Department of Endocrinology and Metabolism, The Third Affiliated Hospital of Sun Yat-sen University, Guangzhou, People's Republic of China; ${ }^{3}$ Department of Neurosurgery, The Third Affiliated Hospital of Sun Yat-sen University, Guangzhou, People's Republic of China; ${ }^{4}$ School of Nursing, Sun Yat-sen University, Guangzhou, People's Republic of China
\end{abstract}

*These authors contributed equally to this work

Correspondence: Cong Ling Department of Neurosurgery, The Third Affiliated Hospital of Sun Yat-sen University, No. 600 Tianhe Road,

Guangzhou, Guangdong, 510630, People's Republic of China

Tel +86-|3580465 I 2 |

Email doctor200@I63.com

Kun Li

School of Nursing, Sun Yat-sen University,

No. 74, Zhongshan Second Road,

Guangzhou, Guangdong, 510085, People's

Republic of China

$\mathrm{Tel}+86-13822206519$

Email likun22@mail.sysu.edu.cn
Objective: The main aim of this study was to evaluate a combined fasting blood glucose based dosage self-titration setting and decision supported telephone coaching intervention on glycemic control and diabetes self-management skills, compared to the conventional care.

Methods: A 12-week, single-blinded, randomized controlled trial was conducted on adults with type 2 diabetes (T2DM) primarily treated with basal insulin. After randomization, the intervention group ( $\mathrm{IG}, \mathrm{n}=426$ ) received a basal insulin self-titration decision support intervention administered by the Diabetes Specialty Nurses while the control group (CG, $\mathrm{n}=423$ ) received conventional care for 12 weeks, both included five telephone interviews. The primary efficacy endpoint was the effect of intervention on glycemic control, measured as the change in glycated hemoglobin (HbA1c) from baseline to Week 12 (after intervention) compared to the control group. Other endpoints included comparisons of the effects of intervention on fasting plasma glucose (FPG), postprandial plasma glucose (PPG), body weight, Michigan diabetes knowledge test (MDKT), diabetes empowerment scale-short Form (DES-DSF), and summary of diabetes self-care activities (SDSCA). Changes in the primary and secondary outcomes were compared using the $t$-test for continuous variables with a normal distribution and $\chi^{2}$-test for categorical variables.

Results: The IG showed more improvements on mean HbA1c, compared to the CG $(-2.8 \%$ vs $-1.8 \%$ ), so did the FPG, PPG, MDKT, DES-DSF and SDSCA (all $\mathrm{P}<0.01$ ) after the 12 -week follow up. Though the final mean insulin dose in the IG was higher than the CG at the end of the study $(0.32 \mathrm{U} / \mathrm{kg}$ vs $0.28 \mathrm{U} / \mathrm{kg})$, the changes of body weight were similar between the two groups ( $0.46 \mathrm{~kg}$ vs $0.40 \mathrm{~kg}, \mathrm{P}=0.246$ ), and the proportion of patients with hypoglycemia events during the whole trial were similar (20.65\% vs $17.73 \%, \mathrm{P}=0.279)$.

Conclusion: Decision supporting of basal insulin glargine self-titration assisted by Diabetes Specialty Nurses is effective and safe in patients with T2DM. Decision supported telephone coaching intervention offers ongoing encouragement, guidance, and determination of relevant sources of decisional conflict, facilitating adjusting the insulin dose.

Keywords: type 2 diabetes mellitus, basal insulin, dosage titration

\section{Introduction}

T2DM has become a major public health challenge in China, because its prevalence has increased from $3.2 \%$ in 1996 to $12.8 \%$ in $2017 .{ }^{1}$ However, according to the national analysis, only $32.2 \%$ patients with T2DM have received appropriate treatment, and the percent of adequate glycemic control is only about $37.6 \%$ for patients 
on insulin therapy, much lower than the patients (50.2\%) on oral antidiabetic drugs (OADs). ${ }^{2}$

For patients with T2DM who have suboptimal glycemic control and show no response to OADs, basal insulin (BI) is one of the first-choice for glucose treatment. ${ }^{3,4}$ Numerous randomized controlled trials (RCTs) and real-life studies, ${ }^{4}$ including data from our country, ${ }^{5,6}$ have validated the efficacy of BI on glycemic control, now the decade of secondgeneration basal insulin analogues has come. ${ }^{7} \mathrm{U}-100$ glargine, the first long-acting insulin analog approved in China, reduces the risk of symptomatic and nocturnal hypoglycemia to a greater extent than NPH insulin in real-life studies from our country. ${ }^{6,8}$ Glargine has been widely used in China for treatment of T2DM, with cost-saving advantage ${ }^{9}$ and acceptable side-effects, such as slight weight gain and low hypoglycemia risk. ${ }^{9,10}$ However, several critical barriers to BI therapy pose a challenge, including suboptimal dose titration and fear of hypoglycemia.

Timely insulin initiation and adequate titration of the insulin dose by either physicians or patients are essential steps to achieve and maintain treatment goals. In a realworld study for T2DM, $43.4 \%$ patients' dose was barely adjusted 6 months after initiation of BI in our country, ${ }^{6}$ in another study the proportion of insulin discontinuation was high within 6 months after initiating BI. ${ }^{11}$ The situation of delayed insulin initiation and suboptimal dose titration in Chinese patients with T2DM is usual, ${ }^{6}$ which could be attributed to limited healthcare resources and clinical inertia among both patients and clinicians. The "clinical inertia" in USA also results in poor glycemic control, it was reported while $>75 \%$ of healthcare professionals discussed titration at the initiation visit, only $16 \%$ to $28 \%$ of patients remembered such discussions, many (32-42\%) were unaware of the need to titrate BI. ${ }^{12}$ In our country, most of the patients not usually see their doctors and nurses after initiation of basal insulin, we even saw a patient full of complain of insulin injection after keeping using $0.5 \mathrm{U} /$ day insulin for several weeks. BI discontinuation significantly associated with hospital level, patient recruitment setting, age, education level, out-of-pocket ratio, et al. ${ }^{11}$ A disconnect exists between doctors and patients perceived barriers to effective BI titration, only patient-led initiation and titration of basal insulin without help from the nurse seemed not enough for glucose control in our country, ${ }^{13}$ so we want to find more effective and practical support tools to assist patients for BI titration.

Patient empowerment promotes self-care and development of innate capacity of responsibility through education and motivation by healthcare professionals. ${ }^{14}$ Health-related decision aids improves knowledge of treatment options, provides realistic expectations of the benefits and risks, and allows choices consistent with values. ${ }^{15}$ Dose titration of glargine based on fasting blood glucose levels using an algorithm is reported as a safe and effective strategy. ${ }^{16}$ Nurse-administered telephone intervention is a wellrecognized approach to improve glycemic control with insulin titration. ${ }^{17,18}$ The purpose of this research was to evaluate the impacts of an fasting blood glucose based dosage titration setting and decision supported telephone coaching intervention by nurses on outpatients with T2DM.

\section{Methods}

\section{Study Design and Setting}

This 12-week, single-blinded, randomized controlled trial was conducted in our department with an intervention group (IG) and a control group (CG). The study protocol was approved by the Ethics Committee of the Third Affiliated Hospital of Sun Yat-sen University. All patients provided written informed consent prior to the participation in the study. After the last interview, the patients in the control group were offered the same education and tools as those in the intervention arm. The study was conducted from January 2017 to July 2018.

\section{Participants and Randomization}

Participants were recruited from the Third Affiliated Hospital of Sun Yat-sen University in China. Both the outpatients and the inpatients were screened. Besides face to face, the patients were invited by telephone to participate into the study and received an offer to meet the investigator. After confirming their intent to participate, patients signed an informed consent form and received the baseline interview.

Inclusion criteria were as follows: $18 \leq$ age $\leq 70$ years, with a diabetes duration of $\geq 2$ years, having used two to three oral antidiabetic drugs (OADs) before screening, $7<\mathrm{HbAlc}$ $<11 \%$, and $20 \leq$ body mass index (BMI) $<30 \mathrm{~kg} / \mathrm{m}^{2}$. Moreover, all patients must agree to perform blood glucose monitoring using a blood glucose meter and completed our follow-up survey and questionnaires. Exclusion criteria were as follows: current or previous use of insulin, acute or severe chronic diabetic complications or illnesses, and positive for glutamic acid decarboxylase antibodies. About four to five patients were enrolled in a day. 
Table I The Similarity and Difference Between the Intervention Group and Control Group

\begin{tabular}{|c|c|c|}
\hline The Care in Our Study & $\begin{array}{l}\text { Intervention Group } \\
(n=436)\end{array}$ & $\begin{array}{l}\text { Control Group } \\
(n=433)\end{array}$ \\
\hline Screening and sign informed consent form & Yes & Yes \\
\hline $\begin{array}{l}\text { Baseline(before randomization) } \\
\text { - Collect demographic and anthropometric data } \\
\text { - Test HbAIc, FPG, and 2hPPG } \\
\text { - Evaluate the score of MDKT, DES-DSF, and SDSCA } \\
\text { - Standard diabetes education (including insulin injection) } \\
\text { - Begin basal insulin glargine treatment }\end{array}$ & Yes & Yes \\
\hline Randomization & Yes & Yes \\
\hline Adjust insulin doses by the doctors' suggestions & Yes & Yes \\
\hline Monitoring fasting glucose per day and random glucose & Yes & Yes \\
\hline Record insulin dose, hypoglycemic events, and other $\mathrm{AE}$ & Yes & Yes \\
\hline $\begin{array}{l}\text { Five coaching calls at the } I, 2,4,8, I 2 \text { weeks by the nurse } \\
\text { - Collect the insulin dose and the AE of the participants } \\
\text { - Guide the Insulin dose titration }\end{array}$ & $\begin{array}{l}\text { Yes } \\
\text { Yes }\end{array}$ & $\begin{array}{l}\text { Yes } \\
\text { No }\end{array}$ \\
\hline Contact the investigator for emergency problems & Yes & Yes \\
\hline $\begin{array}{l}\text { End of the study ( } 12 \text { weeks after randomization) } \\
\text { - Collect demographic and anthropometric data } \\
\text { - Test HbAIc, FPG, and } 2 \text { hPPG } \\
\text { - Evaluate the score of MDKT, DES-DSF, and SDSCA } \\
\text { - Recycle the diary (including the insulin dose, hypoglycemic events and other AE) }\end{array}$ & Yes & Yes \\
\hline
\end{tabular}

Abbreviations: FPG, fasting plasma glucose; PPG, postprandial plasma glucose; MDKT, Michigan diabetes knowledge test; DES-DSF, diabetes empowerment scale-short Form; SDSCA, summary of diabetes self-care activities; AE, adverse events.

After enrollment, all patients were given baseline interview with the responsible doctor and nurse. At baseline, demographic and anthropometric data were recorded. FPG, PPG, and HbAlc were measured. The scores of MDKT, DES-DSF, and SDSCA were evaluated by the nurses. And then they were provided with standard diabetes education by the nurses, regard to diet, exercise, glucose monitoring, insulin injection, and hypoglycemia. They were given basal insulin glargine treatment by the doctor, beginning with $0.25 \mathrm{U} / \mathrm{kg}$ per day. All patients received standard injection guidelines including rotation habits, spacing injections $1 \mathrm{~cm}$ apart, and using a new needle with every injection. After that, a computer-generated simple randomization table assigned patients at a 1:1 ratio to either the intervention or conventional care (control group) arms, the previous OADs (including sulphonylureas/glinides) were continued. The time spent between the enrollment and randomization was 1-2weeks.

Patients were urged to perform glucose monitoring fasting glucose per day and random glucose when they had hypoglycemic symptoms for the duration of the study.
Minor hypoglycemia was defined as symptoms confirmed by a blood glucose concentration $<3.9 \mathrm{mM}$, with prompt recovery after self-administered carbohydrate. Major hypoglycemia was defined as an event requiring the assistance of another person to administer carbohydrate, glucagon, or other resuscitative treatments. The patients were asked to record their insulin dose, hypoglycemic events and other adverse effects (AE) during the study. And they should adjust insulin doses by the doctors' suggestions as the needed, they could see the doctors as usual outpatients when they needed. Glycemic targets were given by the responsible doctor for each patient. All baseline assessments were repeated at the end of the study (Week 12). Patients from both arms were required to bring their blood glucose meters to clinic at the end of the study. Table 1 shows the similarity and differences in care between the two groups.

\section{Nurses}

The Diabetes Specialty Nurses were chosed to finish the study. They must receive standardized training with regard 
to using decision supported system, empowerment model, procedures for intervention before the study. The training was provided through group discussion, role play and lectures. Only qualified nurses were involved in providing the decision support for this study.

A study day was held for participant nurses to review the protocol, randomization schedules, and Clinical Report Forms (CRF), and to be trained in case record, diabetic education, self-dose-adjustment intervention, and followup (including evaluating the scores of MDKT, DES-DSF, and SDSCA) of the patients.

\section{Intervention Group}

The patients in this group received the basal insulin selftitration decision support program. Intervention participants received one baseline in-person dosage setting and decision coaching session to empower adjustment followed by 5 coaching calls at the 1, 2, 4, 8, 12 weeks delivered by the same nurse. Participants were guided towards personalized dosage decisions according to their individual risk factors in every call. A fasting blood glucose based dosage titration protocol was developed to assist with clarifying values for decision options (shown in Table 2).

Telephone follow-up sessions in the five key weeks were conducted by diabetes specialist nurses selected to complete the study. During the coaching calls, titration decisions to achieve personal glycemic targets were discussed and new or modified dosage regimens were recommended, depending

Table 2 Fasting Blood Glucose Based Dosage Titration Protocol

\begin{tabular}{|l|c|}
\hline $\begin{array}{l}\text { Fasting Capillary } \\
\text { Glucose (mmol/L) }\end{array}$ & Glargine Dose Adjustment (U) \\
\hline$\leq 2.8$ & $\begin{array}{c}\text { a } \\
\text { contact the investigator as a emergency } \\
\text { problems }\end{array}$ \\
\hline $2.9-3.8$ & -4 (if total dose $>45 \mathrm{U}$, reduce 10\%) \\
\hline $3.9-4.3$ & -2 (if total dose $>45 \mathrm{U}$, reduce 5\%) \\
\hline $4.4-6.1$ & 0 \\
\hline $6.2-7.8$ & +2 \\
\hline $7.9-9.9$ & +4 \\
\hline$\geq 10$ & +6 \\
\hline
\end{tabular}

Notes: a Dose should be adjusted every 3-4 days to achieve a target range for fasting capillary glucose of 4.4-6. I mmol/L. Change in insulin dose were based on the median of fasting capillary glucose values measured on 3 consecutive days of which the last is the day when titration is to occur on individual circumstances. The optimal insulin glargine dose was defined as that at which patients achieved at least fasting blood glucose values within the predetermined glycemic target. In cases whose glycemic target was not achieved, problem solving for minimizing barriers to goal attainment was implemented, supported by decision coaching for working through relevant decisional conflicts. Detailed notes were written following each coaching call to record blood glucose, insulin dose, dosage titration, and adverse events (including hypoglycemia). In addition to the telephone visits, the patient could contact the investigator for emergency problems.

\section{Control Group}

The patients in this group only adjust their insulin doses based on the doctors' suggestions as needed (seen in Table 2). During the 12-week follow-up, the participants were also followed by 5 coaching calls at the 1, 2, 4, 8, 12 weeks delivered by the same nurse. But the nurses only collected the insulin dose and the adverse effects of the participants, without any suggestions for their insulin dose titration. Detailed notes were also written following each coaching call to record blood glucose, insulin dose, dosage titration, and adverse events (including hypoglycemia). The patient could also contact the investigator for emergency problems.

\section{Study Endpoints}

The primary efficacy endpoint was the effect of intervention on glycemic control, measured as the change in HbAlcfrom baseline to Week 12 (after intervention) compared to the control group. Other endpoints included comparisons of the effects of intervention on FPG, PPG, body weight, Michigan diabetes knowledge test (MDKT), diabetes empowerment scale-short Form (DES-DSF), and summary of diabetes selfcare activities (SDSCA). Tests were translated into Chinese using the academic translation procedure. Outcomes were assessed at baseline and the end of the study (after 12-week follow-up). Safety (including hypoglycemia events) during the study were also assessed.

\section{Instruments and Measures}

Participants completed a questionnaire electronically or on paper at the outpatient clinic before randomization, and at the end of the intervention. It included items on demographics, disease duration and treatment, and 3 validated scales or scores. 
The 23-item Michigan Diabetes Knowledge Test (MDKT) assesses diabetes-related knowledge of patients. The MDKT included 23 items divided into two subscales: the general diabetes subscale with 14 items (items 1-14) (e.g. "Which should not be used to treat low blood glucose?") and the insulin-use subscale with 9 items (items 15-23) (e.g. "If you are sick with the flu, which of the following changes should you make?"). Each item has three or four answer categories, one of which is correct. Higher scores indicated better the knowledge level of the patients. Cronbach's coefficient for general and insulin-use subscales respectively was 0.71 and $0.75 .{ }^{19}$ The Cronbach's coefficient of the Chinese version of MDKT was $0.77 .^{20}$

The 5-item Diabetes Empowerment Scale-Short Form (DES-DSF) assesses individual beliefs regarding management of the psychosocial aspects of diabetes using five response options $(1=$ "never" to $5=$ "always"). The resulting score multiplied by four to obtain standard score (range:20-100points), higher scores indicate better individual beliefs. DES-DSF was one of the questionnaires of the Second Diabetes Attitudes, Wishes and Needs (DAWN2). The questionnaire was translated into the primary local language(s) in 17 countries (USA, Canada, UK, China, etc), then back translated and a harmonization process undertaken to ensure consistency with the original questionnaire, the Cronbach's alpha coefficient of China was $0.68 .^{21}$

The 13-item Summary of Diabetes Self-care Activities Measure (SDSCA) evaluates diabetes self-management by examining six aspects of the regimen (diet, exercise, medication taking, blood glucose testing, foot care, and smoking). Respondents recorded the degree of frequency of performance of various activities over the previous 7 days. Scores for each dimension are calculated as the averages of scores on individual items and range between 0 and 7 , higher scores indicate higher scores indicate better diabetes self-management. Average inter-item correlations within scales were high (mean $=0.47$ ) and test-retest correlations were moderate $($ mean $=0.40){ }^{22}$ The Cronbach's coefficient of the Chinese version of SDSCA was 0.62 to 0.92 , and internal consistency was $1.00 .^{23}$

\section{Statistical Analysis}

The study sample size was calculated using a formula to test the difference between two independent sample proportions. We powered our study based on the primary outcome, glucose control (HbAlc), after adding insulin glargine to OADs for 12 weeks. According to the RCT which compared patient-led and physician-led insulin titration, to observe a minimal clinically difference in $\mathrm{HbA} 1 \mathrm{c}$ change of $0.17 \%$ from China, ${ }^{13}$ twosided $\alpha=0.05$ and $1-\beta=0.90$, allowing for an attrition rate of $20 \%$ from baseline to follow-up, we will need to recruit 112 participants in the intervention group and 112 in the control group, across the study. Baseline characteristics will be summarised using mean and SD for continuous variables, and n (\%) for categorical variables. Baseline characteristics between the two groups will be compared using either the $t$-test (continuous data) or $\chi^{2}$ test/Fisher's exact test (categorical data). Multivariate linear regression was used to assess whether the intervention affected glycemic outcomes, MDKT, DES-DSF, and SDSCA scores while controlling the following potential confounding factors: age, gender, educational level, BMI, fasting C-peptide, numbers and types of OADs, complication, and comorbidities. Changes in the outcomes were compared using the $t$-test for continuous variables with a normal distribution and $\chi^{2}$-test for categorical variables. The significance level was set at 0.05 . All statistical analyses were performed using IBM SPSS, version 25. Significance was defined as two-tailed $\mathrm{P}<0.05$.

\section{Results}

\section{Participant Characteristics}

Overall, 894 participants were enrolled into the study. 869 patients were randomly assigned into two groups: 434 patients in the intervention group and 435 patients in the control group. The reason for screening failures of the 25 patients was $\mathrm{HbAlc}$ values outside the prespecified interval for inclusion (after central laboratory report) or they refused to sign the informed consent form. Finally 849 patients (97.7\%) completed the trial. Three patients in the IG and five patients in the CG discontinued the study due to consent withdrawal, three patients in the IG and four patients in the CG were lost to follow-up, while two patients in the IG and three patients in the CG discontinued for other reasons. A similar proportion of participants in both groups completed the study; 426 (98.2\%) in the IG and and 423 $(97.2 \%)$ in the CG, respectively (detailed data are shown in Figure 1). Demographic and baseline characteristics of patients were not markedly different between the two groups (Table 3). According to patient reported medical 


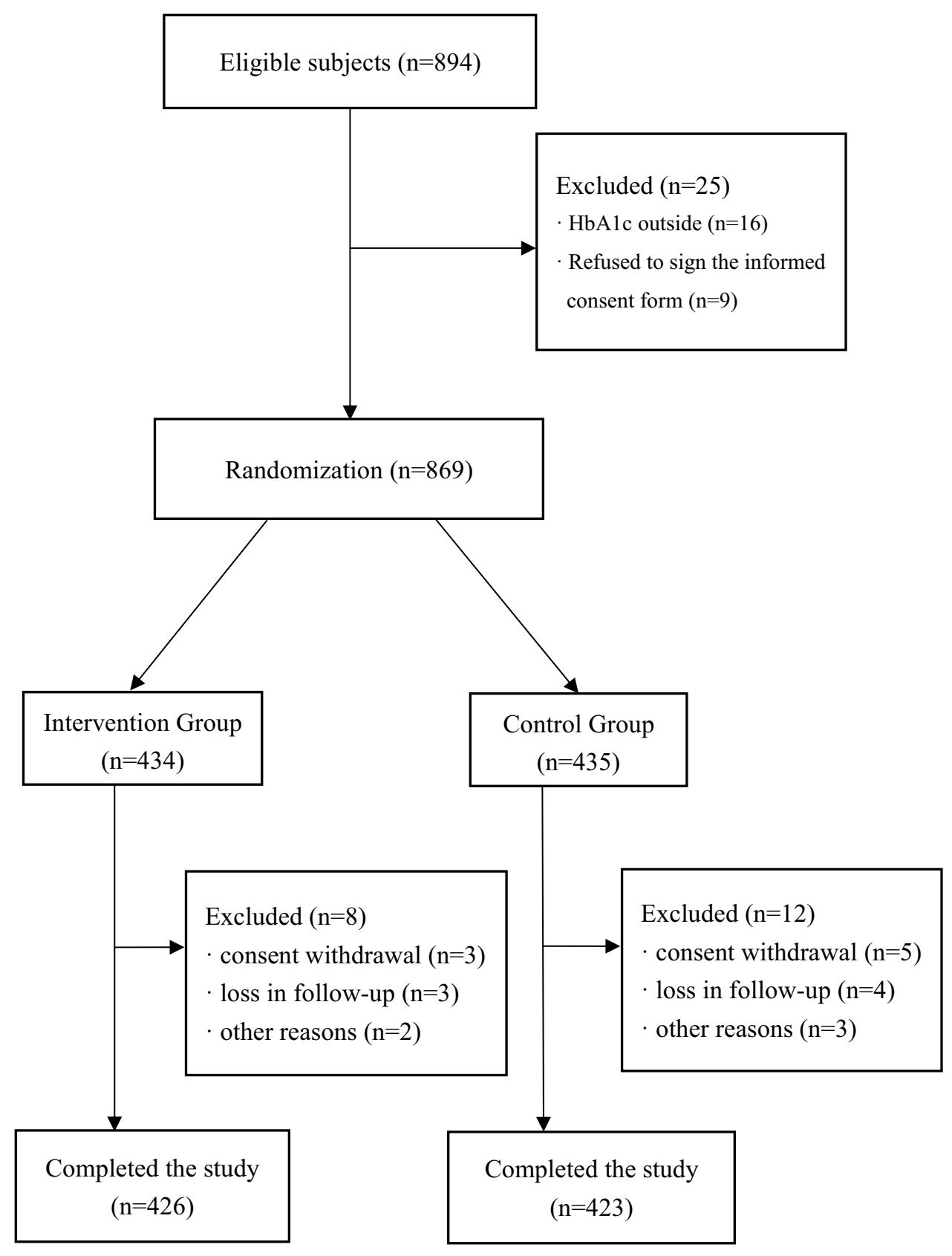

Figure I Trial profile.

history, the most frequent underlying diabetes related comorbidities were: hypertension $(15.9 \%$ vs $17.5 \%$ in the IG and $\mathrm{CG})$ and coronary artery disease $(6.2 \%$ vs $5.0 \%$ in the IG and CG). The previous OADs of the ITT population at baseline are shown in Table 3. Metformin treatment was present (alone or in combination with other agents) in $86.9 \%$ and $83.9 \%$ of patients in the IG and CG. In the IG and CG, the percents in use of $\alpha$ glucosidase inhibitors were $84.3 \%$ and $82.8 \%$, sulfonylureas were $58.8 \%$ and $61.1 \%$, DPP-IV inhibitors were $19.1 \%$ and $21.6 \%$, glinides were $12.9 \%$ and $11.5 \%$, SGLT2 inhibitors were $7.6 \%$ and $9.4 \%$, respectively. The diabetes related complications in the IG and CG are as follows: $27.9 \%$ and $26.4 \%$ for peripheral angiopathy, $17.7 \%$ and $19.0 \%$ for peripheral neuropathy, $13.1 \%$ and $12.0 \%$ for diabetic nephropathy, $7.4 \%$ and $8.3 \%$ for diabetic retinopathy, respectively. Fasting C-peptide levels are also reported in Table 3. 
Table 3 Demographic and Clinical Characteristics at Baseline

\begin{tabular}{|c|c|c|c|}
\hline Characteristics & $\begin{array}{l}\text { Intervention Group } \\
(n=434)\end{array}$ & $\begin{array}{l}\text { Control Group } \\
(n=435)\end{array}$ & $P$-value ${ }^{a}$ \\
\hline \multicolumn{4}{|l|}{ Gender } \\
\hline Male/Female & $263(60.6 \%) / 171(39.4 \%)$ & $248(57 \%) / 187(43 \%)$ & 0.283 \\
\hline Age (years) & $53.62 \pm 14.33$ & $54.77 \pm 13.43$ & 0.224 \\
\hline Duration (years) & $6.39 \pm 5.54$ & $7.88 \pm 6.13$ & 0.139 \\
\hline \multicolumn{4}{|l|}{ Education level (n) } \\
\hline Middle school or lower & $205(47.2 \%)$ & $184(42.3 \%)$ & 0.255 \\
\hline High school & $133(30.6 \%)$ & $137(31.5 \%)$ & \\
\hline College & $96(22.1 \%)$ & $114(26.2 \%)$ & \\
\hline BMI $\left(\mathbf{k g} / \mathbf{m}^{2}\right)$ & $23.5 I \pm 2.25$ & $23.79 \pm 2.72$ & 0.100 \\
\hline Fasting C-peptide (nmol/L) & $0.37 \pm 0.23$ & $0.35 \pm 0.21$ & 0.114 \\
\hline \multicolumn{4}{|l|}{ Numbers of OADs } \\
\hline 2 & $90(20.7 \%)$ & III (25.5\%) & 0.159 \\
\hline 3 & $330(76.0 \%)$ & $324(74.5 \%)$ & \\
\hline \multicolumn{4}{|l|}{ Type of OADs } \\
\hline Metformin & 377 (86.9\%) & $365(83.9 \%)$ & 0.217 \\
\hline$\alpha$-Glucosidase inhibitors & $366(84.3 \%)$ & $360(82.8 \%)$ & 0.532 \\
\hline Sulfonylureas & $255(58.8 \%)$ & $266(61.1 \%)$ & 0.446 \\
\hline DPP-4 inhibitors & $83(19.1 \%)$ & $94(21.6 \%)$ & 0.363 \\
\hline Glinides & $56(12.9 \%)$ & 50 (II.5\%) & 0.526 \\
\hline SGLT2 inhibitors & $33(7.6 \%)$ & $4 \mathrm{l}(9.4 \%)$ & 0.336 \\
\hline \multicolumn{4}{|l|}{ Comorbidity status } \\
\hline Hypertension & 69 (15.9\%) & $76(17.5 \%)$ & 0.534 \\
\hline Coronary artery disease & 27 (6.2\%) & $22(5.0 \%)$ & 0.457 \\
\hline \multicolumn{4}{|l|}{ Diabetic complication } \\
\hline Peripheral angiopathy & I2I (27.9\%) & 115 (26.4\%) & 0.632 \\
\hline Peripheral neuropathy & 77 (I7.7\%) & $83(19.0 \%)$ & 0.611 \\
\hline Diabetic nephropathy & $57(13.1 \%)$ & $52(12.0 \%)$ & 0.600 \\
\hline Diabetic retinopathy & $32(7.4 \%)$ & $36(8.3 \%)$ & 0.620 \\
\hline
\end{tabular}

Notes: Data are expressed as the $\mathrm{N}(\%)$, or mean \pm SD. P-values ${ }^{\mathrm{a}}$ are for comparisons between the two groups.

Abbreviations: OADs, oral antidiabetic drugs; DPP-4, dipeptidyl peptidase-4; SGLT2, sodium-glucose cotransporter 2.

\section{Glycemic Control}

$\mathrm{HbA1c}, \mathrm{FPG}$, and 2hPPG at baseline and end of the intervention are presented in Table 4 . We observed a significant decrease in HbAlc levels in both groups. After 12 weeks of glargine treatment, mean $\mathrm{HbA} 1 \mathrm{c}$ reduction in our patient population was $-2.3 \pm 2.6 \%$. Mean changes in $\mathrm{HbA1c}$ in the intervention group were better than the control group $(-2.8 \pm 1.3 \%$ vs $-1.8 \pm 1.1 \%, \mathrm{P}<0.001)$. The intervention group showed greater reduction of FPG $(-2.63 \pm 1.24 \mathrm{mmol} / \mathrm{L}$ vs1.67 $\pm 1.39 \mathrm{mmol} / \mathrm{L}, \mathrm{P}<0.001)$ and $2 \mathrm{hPPG}(-3.02 \pm 2.31$ vs $2.34 \pm 2.03 \mathrm{mmol} / \mathrm{L}, \mathrm{P}<0.001)$ than the control group (seen in Table 4, both $\mathrm{P}<0.001$ ). We have stratified the post-intervention results in terms of age, sex, educational, diabetic complication, and comorbidity status in Table 5 .

Regarding the rate of target $\mathrm{HbAlc}$ achievement, the proportion of patients achieving glycemic control targets of $\mathrm{HbAlc}<7.0 \%$ and $\leq 6.5 \%$ was higher in the intervention than the control group, by $66.4 \%$ and $45.6 \%$ in the intervention group, and $56.1 \%$ and $35.0 \%$ in the control group (both $\mathrm{P}<0.001$ ).

\section{Scores of MDKT, DES-DSF and SDSCA}

Higher scores in the MDKT $(6.02 \pm 3.86$ vs $3.37 \pm 2.19$, $P<0.001)$, DES-DSF $(38.53 \pm 16.70$ vs $26.00 \pm 18.87$, $P<0.001$ ), and SDSCA (including diet and exercise; all 
Table 4 Glycemic Control

\begin{tabular}{|c|c|c|c|}
\hline Parameter & $\begin{array}{l}\text { Intervention Group } \\
(n=426)\end{array}$ & $\begin{array}{l}\text { Control Group } \\
(n=423)\end{array}$ & ${ }^{\text {a } P}$ value \\
\hline \multicolumn{4}{|l|}{ HbAlc (\%) } \\
\hline Baseline & $9.42 \pm 1.99$ & $9.23 \pm 1.75$ & 0.146 \\
\hline Post-intervention & $6.66 \pm 1.31$ & $7.45 \pm 1.64$ & $<0.001$ \\
\hline Change from baseline & $-2.76 \pm 1.33^{\mathrm{ttt}}$ & $-1.78 \pm 1.14^{t+t}$ & $<0.001$ \\
\hline \multicolumn{4}{|l|}{ FPG (mmol/L) } \\
\hline Baseline & $8.96 \pm 1.98$ & $8.80 \pm 2.12$ & 0.269 \\
\hline Post-intervention & $6.33 \pm 1.21$ & $7.13 \pm 1.83$ & $<0.001$ \\
\hline Change from baseline & $-2.63 \pm 1.24^{t+t}$ & $-1.67 \pm 1.39 t+t$ & $<0.001$ \\
\hline \multicolumn{4}{|l|}{ 2hPPG (mmol/L) } \\
\hline Baseline & $10.73 \pm 2.14$ & $10.52 \pm 1.94$ & 0.117 \\
\hline Post-intervention & $7.72 \pm 1.27$ & $8.18 \pm 1.84$ & $<0.001$ \\
\hline Change from baseline & $-3.02 \pm 2.3 \mathrm{l}^{\mathrm{tt \dagger}}$ & $-2.34 \pm 2.03^{t+t}$ & $<0.001$ \\
\hline
\end{tabular}

Notes: Data are expressed as the mean \pm SD. $\dagger \dagger \dagger$ means $\mathrm{P}<0.00 \mathrm{I}$ compared with the baseline. a $\mathrm{P}$-values are for comparisons between the two groups.

$P<0.001)$ were obtained with the intervention group, compared with the control group (presented in Table 6).

\section{Insulin Doses and Body Weight}

No significant differences were observed between the two groups in terms of dose initiation of insulin glargine (seen in Table 7). The insulin dose for the intervention group was significantly higher than that for the control group at the end of study $(-0.32 \pm 0.12 \mathrm{U} / \mathrm{kg}$ vs $0.28 \pm 0.10 \mathrm{U} / \mathrm{kg}$, shown in Table $7, \mathrm{P}<0.001)$. We observed no significant differences in weight and BMI at baseline between the groups. After treatment, the body weight $(0.46 \pm 0.78 \mathrm{~kg}$ of the IG, $0.40 \pm 0.60 \mathrm{~kg}$ of the IG) and BMI $\left(0.17 \pm 0.31 \mathrm{~kg} / \mathrm{m}^{2}\right.$ of the IG, $0.15 \pm 0.22 \mathrm{~kg} / \mathrm{m}^{2}$ of the $\mathrm{CG})$ of both groups increased slightly, with no significant differences $(\mathrm{P}>0.05)$ between the two groups (seen in Table 7 ).

\section{Safety}

Hypoglycemia rates of both groups at baseline and end of the intervention period are shown in Table 7. Hypoglycemic percentages were similar between the two groups $(20.65 \%$ vs $-17.73 \%$ for the $\mathrm{IG}$ and $\mathrm{CG}, \mathrm{P}=0.279)$. No major hypoglycemic episodes or serious adverse effects (AE) were reported during the experimental period. The most commonly observed AE was upper respiratory tract infection, with no significant differences between the two groups $(12.7 \%$ vs $11.4 \%, \mathrm{P}=0.552$, seen in Table 7$)$.

\section{Discussion}

This study evaluated the efficacy of a combined fasting blood glucose based dosage titration setting and decision supported telephone coaching intervention by the qualified nurses on glycemic control in Chinese patients with T2DM who were treated with insulin glargine. We observed a significant higher reduction (-2.8\%) in HbA1c levels of patients in the IG than those $(-1.8 \%)$ in the CG over 12 weeks of follow-up. There was a study from Asia showed both the patient-led titration and physician-led titration resulted in less mean drop in HbA1c value after $24-$ week use of glargine $(-1.40 \%$ and $-1.25 \%$ ) when compared to our patients in the $\mathrm{IG}^{13}$ Self-managed titration of glargine $300 \mathrm{U} / \mathrm{mL}$ (Gla-300) was superior to physician-managed titration in terms of HbA1c reduction, ${ }^{24}$ both were poorer than that of our study. In the BEYOND study on basal insulin therapy from China, higher proportion of patients achieved HbA1c $<7.0 \%$ in the post-education survey $(36.5 \%$ vs $27.2 \%$ ) with reduced HbA1c levels $(7.72 \%$ vs $8.10 \%){ }^{25}$ These studies showed the better glucose control may due to education. But these data cannot answer whether the improvements benefited from the general education or the insulin dose titration education. An earlier observational study on basal insulin treatment in China reported mean HbA1c levels of $9.6 \%$ at baseline and $7.4 \%$ at 6 months, ${ }^{6}$ the improvements were poorer than our patients in the IG, better than those in the CG. It seems that telephone interview without dosage titration guidance in our study is not effective. The diabetes specialist nurses in the IG kept offering telephone follow-up at key weeks and provide ongoing encouragement, guidance, and support to promote greater treatment efficacy and empowerment for decision changes. The use of decision support to promote 
Table 5 The Post-Intervention Results of HbAlc in Terms of Age, Sex, Educational, Diabetic Complication, and Comorbidity Status

\begin{tabular}{|c|c|c|c|}
\hline Characteristics & $\begin{array}{l}\text { Intervention Group } \\
(n=426)\end{array}$ & $\begin{array}{l}\text { Control Group } \\
(n=423)\end{array}$ & ${ }^{\text {a } P \text {-value }}$ \\
\hline \multicolumn{4}{|l|}{ Gender } \\
\hline Male & $6.66( \pm 1.34)$ & $7.50( \pm 1.67)$ & $<0.001$ \\
\hline Female & $6.64( \pm 1.24)$ & $7.37( \pm 1.60)$ & $<0.001$ \\
\hline \multicolumn{4}{|l|}{ Age (years) } \\
\hline$<60$ & $6.74( \pm I .35)$ & $7.522( \pm 1.68)$ & $<0.001$ \\
\hline$\geq 60$ & $6.54( \pm 1.23)$ & $7.34( \pm 1.60)$ & $<0.001$ \\
\hline \multicolumn{4}{|l|}{ Education level(n) } \\
\hline Middle school or lower & $6.67( \pm 1.32)$ & $7.49( \pm I .70)$ & $<0.001$ \\
\hline High school & $6.77( \pm 1.36)$ & $7.32( \pm|.5|)$ & 0.002 \\
\hline College & $6.48( \pm 1.18)$ & $7.54( \pm|.7|)$ & $<0.001$ \\
\hline \multicolumn{4}{|l|}{ Comorbidity status } \\
\hline \multicolumn{4}{|l|}{ Hypertension } \\
\hline Yes & $6.65( \pm 1.24)$ & $7.87( \pm 1.80)$ & $<0.001$ \\
\hline No & $6.66( \pm 1.32)$ & $7.36( \pm 1.60)$ & $<0.001$ \\
\hline \multicolumn{4}{|l|}{ Coronary artery disease } \\
\hline Yes & $6.57( \pm 1.08)$ & $6.57( \pm 1.08)$ & 0.005 \\
\hline No & $6.66( \pm 1.32)$ & $6.66( \pm 1.32)$ & $<0.001$ \\
\hline \multicolumn{4}{|l|}{ Diabetic complication } \\
\hline \multicolumn{4}{|l|}{ Peripheral angiopathy } \\
\hline Yes & $6.61( \pm 1.23)$ & $7.64( \pm \mid .5 I)$ & $<0.001$ \\
\hline No & $6.68( \pm 1.33)$ & $7.38( \pm 1.68)$ & $<0.001$ \\
\hline \multicolumn{4}{|l|}{ Peripheral neuropathy } \\
\hline Yes & $6.73( \pm I .42)$ & $7.44( \pm 1.83)$ & 0.008 \\
\hline No & $6.64( \pm I .28)$ & $7.45( \pm 1.60)$ & $<0.001$ \\
\hline \multicolumn{4}{|l|}{ Diabetic nephropathy } \\
\hline Yes & $6.66( \pm 1.19)$ & $7.24( \pm 1.54)$ & 0.031 \\
\hline No & $6.66( \pm 1.32)$ & $7.48( \pm 1.66)$ & $<0.001$ \\
\hline \multicolumn{4}{|l|}{ Diabetic retinopathy } \\
\hline Yes & $6.53( \pm 1.38)$ & $7.57( \pm 1.73)$ & 0.008 \\
\hline No & $6.67( \pm 1.30)$ & $7.44( \pm 1.64)$ & $<0.001$ \\
\hline
\end{tabular}

Notes: Data are expressed as mean \pm SD. ${ }^{\text {aP }}$-values are for comparisons between the two groups.

patients' compliance to insulin dose titration is considered an innovative and highly effective strategy for improvement of glycemic control.

Many patients with T2DM continue to achieve suboptimal glycemic control after accepting the BI. ${ }^{6}$ Once basal insulin is initiated, dose titration is an important factor affecting achievement of target glycemic control. ${ }^{26}$ Nurse practitioners, physician assistants, pharmacists, and certified diabetes educators play increasingly valuable roles in terms of insulin initiation and titration. ${ }^{27}$ Incorporation of the services of nurse consultant experts is the key for successful patient treatment with BI, both during hospital stay and after discharge. ${ }^{28}$ In the Italian Titration Approach Study (ITAS) with insulin Gla-300 in insulinnaïve T2DM, when they wanted to compared the glucose control managed by the patient versus the physician, "nurse assisted" was needed in the patient management group. ${ }^{17}$ Involvement of nurses as educators seems very useful in BI treatment. In accordance with previous studies, ${ }^{13}$ the hypoglycemic events and AE in our study 
Table 6 Scores of MDKT, DES-DSF, and SDSCA

\begin{tabular}{|c|c|c|c|}
\hline Score & $\begin{array}{l}\text { Intervention Group } \\
(n=426) \text { Mean } \pm \text { SD }\end{array}$ & $\begin{array}{l}\text { Control Group } \\
(n=423) \text { Mean } \pm \text { SD }\end{array}$ & $P$-value ${ }^{a}$ \\
\hline \multicolumn{4}{|l|}{ MDKT } \\
\hline Baseline & $13.13 \pm 4.08$ & $13.35 \pm 3.89$ & 0.430 \\
\hline Post-intervention & $19.15 \pm 2.64^{\dagger \dagger \dagger}$ & $16.71 \pm 3.72^{\dagger \dagger \dagger}$ & $<0.001$ \\
\hline \multicolumn{4}{|l|}{ DES-DSF } \\
\hline Baseline & $34.00 \pm 12.30$ & $34.06 \pm 16.19$ & 0.950 \\
\hline Post-intervention & $38.53 \pm 16.70^{\dagger t \dagger}$ & $26.00 \pm 18.8^{t+\dagger}$ & $<0.001$ \\
\hline \multicolumn{4}{|l|}{ SDSCA } \\
\hline \multicolumn{4}{|l|}{ Diet } \\
\hline Baseline & $4.69 \pm 1.56$ & $4.80 \pm 1.35$ & 0.275 \\
\hline Post-intervention & $6.26 \pm 0.93^{t+\dagger}$ & $5.61 \pm 1.20^{\dagger t \dagger}$ & $<0.001$ \\
\hline \multicolumn{4}{|l|}{ Exercise } \\
\hline Baseline & $3.56 \pm 1.75$ & $3.46 \pm 1.73$ & 0.413 \\
\hline Post-intervention & $5.27 \pm 1.17^{\dagger+\dagger}$ & $4.19 \pm 1.43^{t+\dagger}$ & $<0.001$ \\
\hline \multicolumn{4}{|c|}{ Blood glucose testing } \\
\hline Baseline & $3.23 \pm 1.68$ & $3.33 \pm 1.71$ & 0.275 \\
\hline Post-intervention & $5.20 \pm 1.15^{\dagger \dagger \dagger}$ & $4.14 \pm 1.49^{\dagger t \dagger}$ & $<0.001$ \\
\hline \multicolumn{4}{|l|}{ Foot care } \\
\hline Baseline & $3.37 \pm 1.59$ & $3.44 \pm 1.68$ & 0.527 \\
\hline Post-intervention & $5.30 \pm 1.14^{\dagger \dagger \dagger}$ & $4.12 \pm 1.44^{t+\dagger}$ & $<0.001$ \\
\hline \multicolumn{4}{|c|}{ Medication taking } \\
\hline Baseline & $5.75 \pm 1.02$ & $5.81 \pm 1.06$ & 0.387 \\
\hline Post-intervention & $6.88 \pm 0.34^{t t \dagger}$ & $6.24 \pm 0.90^{t+\dagger}$ & $<0.001$ \\
\hline \multicolumn{4}{|l|}{ Smoking } \\
\hline Baseline & $3.35 \pm 1.65$ & $3.34 \pm 1.68$ & 0.902 \\
\hline Post-intervention & $5.20 \pm 1.09^{\dagger+t}$ & $4.25 \pm 1.39^{t+t}$ & $<0.001$ \\
\hline
\end{tabular}

Notes: Data are expressed as the mean \pm SD. $\nmid \dagger \dagger$ means $\mathrm{P}<0.00 \mathrm{I}$ compared with the baseline. $\mathrm{P}$ values ${ }^{\mathrm{a}}$ are for comparisons between the two groups.

are not serious in the titration of BI. Our study demonstrated insulin dose titration by the patient, assisted by the nurse, is effective and useful.

The intervention group reported more improvements in diabetes knowledge in our study. Like other developing country, ${ }^{29}$ knowledge on diabetes in patients needs to be changed in our country. In a cross-sectional study in Shanghai, patients with higher knowledge scores were more likely to achieve the combined target goal. ${ }^{30}$ In addition, improvement in empowerment and selfmanagement (including diet, exercise, and blood glucose monitoring) of patients in the IG may contribute to better glucose control. Recent studies have shown that implementation of empowerment strategies among individuals with diabetes was associated with improvements in $\mathrm{HbAlc}$ levels, self-management, and distress. ${ }^{31,32}$ In nationwide survey, Chinese patients with diabetes education achieved better glycemic control than un-educated patients. ${ }^{33}$ In an outlying city of China, nutritional and eating education also make use. ${ }^{34}$ Patient understanding of diabetes selfmanagement is critical to optimize safety and enhance outcomes. ${ }^{35}$ Based on our result and our diabetes education school, we will try to train nurses from different cities and rural areas to be qualified for the education skills that were used in our study. These nurses will contribute to better glucose control of T2DM, in terms of the health system structure in China.

There are some limitations in this study. First, this study was conducted at a single center. Second, although sample size was sufficient to assess primary outcomes, some information of the participants are missing, such as the income level. Third, the 12-week intervention period may not have been sufficient to address maintenance of implemented changes. Nevertheless, our results clearly suggest that decision support of self-titration of basal insulin in outpatients with T2DM is an effective and safe strategy. Further research 
Table 7 Insulin Dose, Body Weight, and Safety

\begin{tabular}{|c|c|c|c|}
\hline Parameter & $\begin{array}{l}\text { Intervention Group }(n=426) \\
N(\%) \text { or Mean } \pm \text { SD }\end{array}$ & $\begin{array}{l}\text { Control Group }(n=423) \\
N(\%) \text { or Mean } \pm \text { SD }\end{array}$ & $P$-value ${ }^{a}$ \\
\hline \multicolumn{4}{|l|}{ Insulin dose (U/kg) } \\
\hline Baseline & $0.25 \pm 0.11$ & $0.25 \pm 0.10$ & 0.983 \\
\hline Post-intervention & $0.32 \pm 0.12^{t+t}$ & $0.28 \pm 0.10^{\dagger+\dagger}$ & $<0.001$ \\
\hline \multicolumn{4}{|l|}{ Weight (kg) } \\
\hline Baseline & $63.2 \pm 8.6$ & $64.3 \pm 9.3$ & 0.070 \\
\hline Post-intervention & $63.62 \pm 8.42$ & $64.68 \pm 9.17$ & 0.081 \\
\hline Change from baseline & $0.46 \pm 0.78^{t+t}$ & $0.40 \pm 0.60^{t+t}$ & 0.242 \\
\hline \multicolumn{4}{|l|}{ BMI $\left(\mathrm{kg} / \mathrm{m}^{2}\right)$} \\
\hline Baseline & $23.5 \pm 2.3$ & $23.8 \pm 2.7$ & 0.111 \\
\hline Post-intervention & $23.69 \pm 2.21$ & $23.93 \pm 2.71$ & 0.141 \\
\hline Change from baseline & $0.17 \pm 0.3 \mathrm{I}^{\mathrm{tt \dagger}}$ & $0.15 \pm 0.22^{\dagger+\dagger}$ & 0.181 \\
\hline \multicolumn{4}{|l|}{ Hypoglycemia (\%) } \\
\hline Week 0-4 & $16(3.76)$ & $13(3.07)$ & 0.584 \\
\hline Week 5-8 & $34(7.98)$ & $29(6.86)$ & 0.532 \\
\hline Week 9-12 & $38(8.92)$ & $33(7.80)$ & 0.556 \\
\hline Total & $88(20.65)$ & $75(17.73)$ & 0.279 \\
\hline \multicolumn{4}{|l|}{ URTI (\%) } \\
\hline Week 0-12 & $54(12.7)$ & $48(11.4)$ & 0.552 \\
\hline
\end{tabular}

Notes: Data are expressed as the $\mathrm{N}(\%)$, or mean $\pm \mathrm{SD}$. $\dagger \dagger \dagger$ means $\mathrm{P}<0.00 \mathrm{I}$ compared with the baseline. $\mathrm{P}$-values ${ }^{\mathrm{a}}$ are for comparisons between the two groups. Abbreviations: BMI, body mass index; URTI, upper respiratory tract infection.

with longer-term follow-up of patients and collected more detailed data that can be stratified, could be conducted to validate the efficacy of our decision supported intervention.

\section{Conclusions}

A combined fasting blood glucose based dosage titration setting and decision supported telephone coaching intervention by diabetes specialist nurses is effective in improving glycemic control with insulin glargine. Patients with T2DM need continued decision supporting, including adequate glycemic target levels and more active insulin dose titration after initiation of insulin therapy.

\section{Impact Statement}

The study developed a fasting blood glucose based dosage titration protocol which can assist with clarifying values for decision options.

\section{Data Sharing Statement}

The authors Cong Ling and Kun Li can be contacted for the data of this study for the same.

\section{Ethics Approval}

The study protocol was approved by the Ethics Committee of the Third Affiliated Hospital of Sun Yat-sen University ([2016]2-12). We confirm that this study was conducted in accordance with the Declaration of Helsinki. All patients provided written informed consent prior to participation in the study. The trial was registered at https://clinicaltrials.gov/, with the NCT number (NCT02526810).

\section{Funding}

This study was supported by Guangdong Basic and Applied Basic Research Foundation(2020A1515110195) and Chinese Nursing Association(ZHKY202023).

\section{Disclosure}

The authors declare that there is no conflict of interest.

\section{References}

1. Li Y, Teng D, Shi X, et al. Prevalence of diabetes recorded in mainland China using 2018 diagnostic criteria from the American Diabetes Association: national cross sectional study. BMJ. 2020;369:m997. doi:10.1136/bmj.m997

2. Wang L, Gao P, Zhang M, et al. Prevalence and ethnic pattern of diabetes and prediabetes in China in 2013. JAMA. 2017;317 (24):2515-2523. doi:10.1001/jama.2017.7596 
3. Jia W, Weng J, Zhu D, et al. Standards of medical care for type 2 diabetes in China 2019. Diabetes Metab Res Rev. 2019;35(6):e3158. doi: $10.1002 / \mathrm{dmrr} .3158$

4. American Diabetes Association. 9. pharmacologic approaches to glycemic treatment: standards of medical care in diabetes-2021. Diabetes Care. 2021;44(Supp11):S111-S124. doi:10.2337/dc21-S009

5. Lin S, Chen M, Chen W, et al. A randomized trial of insulin glargine plus oral hypoglycemic agents versus continuous subcutaneous insulin infusion to treat newly diagnosed type 2 diabetes. J Diabetes Res. 2018;2018:2791584. doi:10.1155/2018/2791584

6. Ji L, Zhang P, Zhu D, et al. Observational Registry of Basal Insulin Treatment (ORBIT) in patients with type 2 diabetes uncontrolled with oral antihyperglycaemic drugs: real-life use of basal insulin in China. Diabetes Obes Metab. 2017;19(6):822-830. doi:10.1111/dom.12886

7. Cheng A, Bailey TS, Mauricio D, Roussel R. Insulin glargine 300 U/ $\mathrm{mL}$ and insulin degludec: a review of the current evidence comparing these two second-generation basal insulin analogues. Diabetes Metab Res Rev. 2020;e3329. doi:10.1002/dmrr.3329

8. Zhang T, Ji L, Gao Y, et al. Observational registry of basal insulin treatment in patients with type 2 diabetes in China: safety and hypoglycemia predictors. Diabetes Technol Ther. 2017;19 (11):675-684. doi:10.1089/dia.2017.0111

9. Su W, Li C, Zhang L, Lin Z, Tan J, Xuan J. Meta-analysis and cost-effectiveness analysis of insulin glargine $100 \mathrm{U} / \mathrm{mL}$ versus insulin degludec for the treatment of type 2 diabetes in China. Diabetes Ther. 2019;10(5):1969-1984. doi:10.1007/s13300-019-00683-2

10. Tien KJ, Hung YJ, Chen JF, et al. Basal insulin therapy: unmet medical needs in Asia and the new insulin glargine in diabetes treatment. J Diabetes Investig. 2019;10(3):560-570. doi:10.1111/ jdi. 12984

11. Zhang P, Zhang H, Li X, Chen M, Wang D, Ji L. Factors and outcomes associated with discontinuation of basal insulin therapy in patients with type 2 diabetes mellitus. Endocrinol Diabetes Metab. 2020;3(2):e122. doi:10.1002/edm2.122

12. Berard L, Bonnemaire M, Mical M, Edelman S. Insights into optimal basal insulin titration in type 2 diabetes: results of a quantitative survey. Diabetes Obes Metab. 2018;20(2):301-308. doi:10.1111/ dom.13064

13. Garg SK, Admane K, Freemantle N, et al. Patient-led versus physician-led titration of insulin glargine in patients with uncontrolled type 2 diabetes: a randomized multinational ATLAS study. Endocr Pract. 2015;21(2):143-157. doi:10.4158/EP14079.OR

14. Gómez-Velasco DV, Almeda-Valdes P, Martagón AJ, Galán-Ramírez GA, Aguilar-Salinas CA. Empowerment of patients with type 2 diabetes: current perspectives. Diabetes Metab Syndr Obes. 2019;12:1311-1321. doi:10.2147/DMSO.S174910

15. Stacey D, Légaré F, Lewis K, et al. Decision aids for people facing health treatment or screening decisions. Cochrane Database Syst Rev. 2017;4(4):D1431. doi:10.1002/14651858.CD001431.pub5

16. Misra A, Patel M, Agarwal P, et al. Effectiveness and safety of physician-led versus patient-led titration of insulin glargine in indian patients with type 2 diabetes mellitus: a subanalysis of the Asian Treat to Target Lantus Study (ATLAS). Diabetes Technol Ther. 2019;21(11):656-664. doi:10.1089/dia.2019.0037

17. Bonadonna RC, Giaccari A, Buzzetti R, et al. Italian Titration Approach Study (ITAS) with insulin glargine $300 \mathrm{U} / \mathrm{mL}$ in insulin-naive type 2 diabetes: design and population. Nutr Metab Cardiovasc Dis. 2019;29(5):496-503. doi:10.1016/j. numecd.2019.01.011

18. Bellido V, Bellido D, Tejera C, et al. Effect of telephone-delivered interventions on glycemic control in type 2 diabetes treated with glargine insulin. Telemed $J$ E Health. 2019;25(6):471-476. doi:10.1089/tmj.2018.0014

19. Fitzgerald JT, Funnell MM, Hess GE, et al. The reliability and validity of a brief diabetes knowledge test. Diabetes Care. 1998;21 (5):706-710. doi:10.2337/diacare.21.5.706
20. Sn S, Wg Z, Yy D. The current status and influential factors of self-management in diabetic patients. Chin $J$ Nurs. 2011;3 (46):229-233.

21. Nicolucci A, Kovacs BK, Holt RI, et al. Diabetes attitudes, wishes and needs second study (DAWN2 ${ }^{\mathrm{TM}}$ ): cross-national benchmarking of diabetes-related psychosocial outcomes for people with diabetes. Diabet Med. 2013;30(7):767-777. doi:10.1111/dme.12245

22. Toobert DJ, Hampson SE, Glasgow RE. The summary of diabetes self-care activities measure: results from 7 studies and a revised scale. Diabetes Care. 2000;23(7):943-950. doi:10.2337/ diacare.23.7.943

23. Al C, Zl Z, Zh L. Self-management and quality of life in patients with diabetes mellitus. Chin J Behav Med Sci. 2006;15:434-436.

24. Russell-Jones D, Dauchy A, Delgado E, et al. Take control: a randomized trial evaluating the efficacy and safety of self- versus physician-managed titration of insulin glargine $300 \mathrm{U} / \mathrm{mL}$ in patients with uncontrolled type 2 diabetes. Diabetes Obes Metab. 2019;21 (7):1615-1624. doi:10.1111/dom.13697

25. Weng J, Zhao J, Zhou Z, et al. Observational study evaluating the effectiveness of physician-targeted education for improving glycemic management of patients with type 2 diabetes (BEYOND II). J Diabetes. 2020;12(1):66-76. doi:10.1111/1753-0407.12963

26. Mocarski M, Yeaw J, Divino V, et al. Slow titration and delayed intensification of basal insulin among patients with type 2 diabetes. J Manag Care Spec Pharm. 2018;24(4):390-400. doi:10.18553/ jmcp.2017.17218

27. María MM, Ramos A, Martínez RM, Pujol GI, Pérez A. Implementation of an inpatient hyperglycemia management program incorporating a nurse consultant. Endocrinol Diabetes Nutr. 2020;67 (7):461-468. doi:10.1016/j.endinu.2019.07.010

28. Rogers E, Aidasani SR, Friedes R, et al. Barriers and facilitators to the implementation of a mobile insulin titration intervention for patients with uncontrolled diabetes: a qualitative analysis. Jmir Mhealth Uhealth. 2019;7(7):e13906. doi:10.2196/13906

29. Chawla SPS, Kaur S, Bharti A, et al. Impact of health education on knowledge, attitude, practices and glycemic control in type 2 diabetes mellitus. J Family Med Prim Care. 2019;8(1):261-268. doi:10.4103/ jfmpc.jfmpc_228_18

30. Yang H, Gao J, Ren L, et al. Association between knowledge-attitude-practices and control of blood glucose, blood pressure, and blood lipids in patients with type 2 diabetes in Shanghai, China: a Cross-Sectional Study. $J$ Diabetes Res. 2017;2017:3901392. doi:10.1155/2017/3901392

31. Baldoni NR, Aquino JA, Sanches-Giraud C, et al. Collective empowerment strategies for patients with diabetes mellitus: a systematic review and meta-analysis. Prim Care Diabetes. 2017;11 (2):201-211. doi:10.1016/j.pcd.2016.09.006

32. Swoboda CM, Miller CK, Wills CE. Impact of a goal setting and decision support telephone coaching intervention on diet, psychosocial, and decision outcomes among people with type 2 diabetes. Patient Educ Couns. 2017;100(7):1367-1373. doi:10.1016/j. pec.2017.02.007

33. Guo XH, Yuan L, Lou QQ, et al. A nationwide survey of diabetes education, self-management and glycemic control in patients with type 2 diabetes in China. Chin Med J. 2012;125(23):4175-4180.

34. Wang H, Song Z, Ba Y, Zhu L, Wen Y. Nutritional and eating education improves knowledge and practice of patients with type 2 diabetes concerning dietary intake and blood glucose control in an outlying city of China. Public Health Nutr. 2014;17(10):2351-2358. doi:10.1017/S1368980013002735

35. Liu XL, Wu CJ, Willis K, Shi Y, Johnson M. The impact of inpatient education on self-management for patients with acute coronary syndrome and type 2 diabetes mellitus: a cross-sectional study in China. Health Educ Res. 2018;33(5):389-401. doi:10.1093/her/cyy023 


\section{Publish your work in this journal}

Diabetes, Metabolic Syndrome and Obesity: Targets and Therapy is an international, peer-reviewed open-access journal committed to the rapid publication of the latest laboratory and clinical findings in the fields of diabetes, metabolic syndrome and obesity research. Original research, review, case reports, hypothesis formation, expert opinion and commentaries are all considered for publication. The manuscript management system is completely online and includes a very quick and fair peer-review system, which is all easy to use. Visit http://www.dovepress.com/testimonials.php to read real quotes from published authors.

Submit your manuscript here: https://www.dovepress.com/diabetes-metabolic-syndrome-and-obesity-targets-and-therapy-journal 